\title{
Percepción de la pérdida auditiva en trabajadores del sector de la construcción, ciudad Quito
}

\section{Perception of hearing loss in workers in the construction sector, Quito city}

Ing. Gloria Estefanía Vásquez Larriba

Ph. D Yolis Yajaira Campos Villalta

Ph. D Antonio Ramón Gómez García

Universidad Internacional SEK Ecuador, Ecuador

Ph. D Luis Guillermo Vásquez Zamora

Universidad Internacional del Ecuador, Ecuador

Universidad San Gregorio de Portoviejo, Ecuador

Autor para correspondencia: loryvasquez@ hotmail.com, yolis.campos@uisek.edu.ec, luisvasquezzamora@hotmail.com,_antonio.gomez@uisek.edu.ec

Fecha de recepción: 12 de Junio de 2017 - Fecha de aceptación: 15 de Noviembre de 2017

Resumen: Son limitados los estudios respecto a conductas de los trabajadores frente a los riesgos laborales a los cuales están expuestos, es por ello que el estudio de estos es un factor relevante al momento de la prevención de riesgos pues no basta con las medidas preventivas de origen técnico y organizativo, es necesario el estudio del factor humano y su fiabilidad. El objetivo del estudio fue conocer la percepción de los trabajadores expuestos a ruido frente a la pérdida auditiva y uso de protección auditiva. Se realizó un estudio descriptivo de corte transversal a 20 trabajadores del área de producción del sector construcción, expuestos a un nivel de ruido continuo diario mayor o igual a 85 decibelios (A) en su jornada. El total de trabajadores percibieron como no problemático el daño generado por la exposición al ruido en su trabajo diario sin embargo todos estaban conscientes que la exposición a ruido fuerte puede causar daños a su audición. El 85\% de trabajadores manifestaron que para mantener su audición era importante usar protectores auditivos cuando tienen exposición a ruidos por encima del límite permitido. Los trabajadores expresaron que no siempre pueden decidir cuándo necesitan cambiar los protectores auditivos. El estudio realizado evidencio que, aunque los trabajadores tienen conocimiento respecto a los efectos ocasionados por ruido excesivo y la importancia del uso de protectores auditivos, se requiere mayor entrenamiento en autosuficiencia para el uso, mantenimiento y sustitución de los protectores auditivos; así como la dotación de equipos más ergonómicos.

Palabras clave: actitud; ruido; pérdida auditiva

Abstract: Studies are limited on the behavior of workers against the occupational hazards to which they are exposed, which is why the study of these is a relevant factor at the time of risk prevention because it is not enough with preventive measures of technical origin and organizational, it is necessary to study the human factor and its reliability. The objective of the study was to know the perception of workers exposed to noise in the face of hearing loss and hearing protection. A cross - sectional descriptive study was carried out on 20 workers in the production area of the construction sector, exposed to a continuous daily noise level equivalent to or greater than 85 
decibels (A) in their workday. The total number of workers perceived the damage generated by exposure to noise in their daily work as non-problematic, however, all workers were aware that exposure to loud noise can cause damage to their hearing. $85 \%$ of workers stated that it was important to wear hearing protectors when they had exposure to noise above the allowed limit to maintain their hearing. Workers said they can't always decide when they need to change hearing protectors. The study showed that, although workers are aware of the effects caused by excessive noise and the importance of the use of hearing protectors, greater training in self-sufficiency is required for the use, maintenance and replacement of hearing protectors; As well as the provision of more ergonomic equipment.

Key words: attitude; noise; hearing loss

\section{Introducción}

Aproximadamente entre el 7 al 21\% de las pérdidas de agudeza auditiva está causadas por el ruido industrial (Reddy et al., 2012).

Son escasos los estudios realizados en Ecuador que aporten información sobre las conductas de los trabajadores frente a los riesgos laborales a los que se encuentran expuestos, permitiendo conocer la influencia de la percepción del trabajador frente a la exposición continuada de un riesgo laboral y, por tanto, la probabilidad de sufrir un accidente o contraer una enfermedad (Gómez et al., 2013).

Como bien indica Meliá (2007), el comportamiento depende de cadenas de decisiones, es decir, la percepción que tenga el trabajador influye en el comportamiento de éste ante diferentes situaciones y actividades, las cuales, no están exentas de riesgos que pueden desencadenar en accidentes de trabajo y en la aparición de enfermedades profesionales, independientemente de las medidas preventivas implantadas en la organización (Gómez et al., 2013).

No basta considerar solamente las medidas preventivas de tipo técnico u organizativo, sino que, el factor humano y su fiabilidad son aspectos fundamentales que deben ser considerados para una adecuada y correcta gestión de la seguridad y salud ocupacional.

Desde la percepción de los trabajadores frente a exposición, los riesgos presentes en el lugar de trabajo, Morillejo (2002), considera que la percepción del riesgo en el ámbito laboral se convierte en un elemento crucial para entender las prácticas de trabajo inseguras de los trabajadores.

En este sentido, cuando se evalúa el riesgo laboral entran en juego diferentes creencias, valores, emociones y actitudes del trabajador frente al mismo, por tanto, la percepción es multidimensional: influenciada por connotaciones sociales, culturales y psicológicos (Contreras, 2013).

El objetivo del presente estudio fue conocer la percepción de los trabajadores expuestos a ruido frente a la pérdida auditiva y uso de protección auditiva.

\section{Material - métodos}


Estudio descriptivo de corte transversal realizado en enero de 2017 a 20 trabajadores (hombres entre 27 a 40 años de edad) del área de producción del sector construcción, expuestos a un nivel de ruido continuo diario $\geq 85$ decibelios (A) en su jornada laboral y con una antigüedad en la actividad superior a los 5 años.

En función al objeto de estudio y al igual que otros estudios (Svensson et al., 2004; Hannah et al, 2015), se aplicó para la recogida de información el cuestionario sobre Creencias y Actitudes sobre la Protección Auditiva y Pérdida de Audición (Kepplet at al, 2015) (en inglés Beliefs About Hearing Protection and Hearing Loss - BAHPHL) desarrollado y validado por el Instituto Nacional para la Seguridad y Salud Ocupacional del Departamento de Trabajo de los EE.UU. (National Institute for Occupational Safety and Health - NIOSH), permitiendo conocer la percepción de los trabajadores del riesgo que representa estar expuesto a altos niveles de ruido en el lugar de trabajo.

El cuestionario aplicado se compone de 28 preguntas agrupadas en las siguientes dimensiones; Susceptibilidad pérdida auditiva (Preguntas 1 y 14), Consecuencias pérdida auditiva (Preguntas 2 y 13), Beneficios acciones preventivas (Preguntas 5, 16 y 24), Acción preventiva - comodidad (Preguntas 6, 17, 19 y 25), Acción preventiva - silenciar (Preguntas 7, 8, 18 y 26), Comportamientos (Preguntas 9, 10, 21, 22, 27 y 28), Normas sociales (Preguntas 3, 11 y 20) y Autoeficacia (Preguntas 4, 12, 15 y 23), permitiendo conocer las actitudes y creencias de los trabajadores frente a la pérdida auditiva.

Las respuestas para cada pregunta se distribuyen con una escala tipo Likert; 0) Ni de acuerdo ni en desacuerdo, 1) Totalmente de acuerdo, 2) Estoy de acuerdo, 3) En desacuerdo y 4) Totalmente en desacuerdo.

El cuestionario fue auto-administrado por el Departamento de Seguridad y Salud Ocupacional de la empresa constructora, los trabajadores encuestados fueron informados sobre los objetivos, el consentimiento verbal a participar voluntariamente y el carácter anónimo de la misma. Se aclararon las preguntas y respuestas de la encuesta.

Recopilados los datos se procedió al control de calidad para garantizar la autenticidad de los mismos.

Todos los cuestionarios se registraron en una base de datos Microsoft Excel y fueron analizados mediante Statistical Package for the Social Sciences (versión 24). Para el análisis estadístico se emplearon las frecuencias absolutas y relativas.

\section{Resultados}

En la Tabla 1 se presenta la distribución de los resultados en función a cada una de las dimensiones del cuestionario de creencias, sobre la protección auditiva y la pérdida auditiva en los trabajadores encuestados.

\section{Susceptibilidad de pérdida auditiva}


El 80\% de los trabajadores encuestados manifestaron estar totalmente en desacuerdo y/o en desacuerdo en lo referente a trabajar próximos a fuentes de ruido sin llegar a causarles un daño a la salud. El total de trabajadores perciben como no problemático el daño generado por la exposición al ruido en su trabajo diario.

\section{Consecuencias de la pérdida auditiva}

El total de los trabajadores encuestados consideran que existiría mayor dificultad para comunicarse cuando se haya generado un daño en su audición. El 95\% de los trabajadores manifestaron estar conscientes que la exposición a ruido fuerte puede causar daños a su audición.

\section{Beneficios acciones preventivas}

La mayoría de los trabajadores $(85 \%)$ de la población encuestada están convencidos que para poder mantener su audición es importante usar protectores auditivos cuando se encuentran expuestos a ruidos por encima del límite permitido.

\section{Acción preventiva - comodidad}

Un $80 \%$ de las personas que fueron encuestadas expresaron estar totalmente de acuerdo y/o de acuerdo con el hecho de que encuentran incomodo el uso de orejeras como medio de protección auditiva debido a que estas proporcionan calor, tienen un peso considerable y ejercen presión en los oídos. Sin embargo, la mayoría de los encuestados consideran que al ajustar correctamente los protectores auditivos se puede obtener la comodidad necesaria.

\section{Acción preventiva - silenciar}

A un 35\% de los colaboradores les resulta difícil escuchar las señales sonoras de alarma cuando están usando protectores auditivos en contraste con un 55\% los cuales están en desacuerdo con esta afirmación.

\section{Comportamientos}

Un $90 \%$ de la población de trabajadores encuestados tiene la intención de utilizar protectores auditivos cuando se encuentren próximos a herramientas o equipos que generen ruido considerable. De igual manera se pudo evidenciar de acuerdo a los datos analizados que la mayoría de estos trabajadores y sus compañeros usan protectores auditivos cuando trabajan cerca de fuentes de ruido fuerte.

\section{Normas sociales}

De acuerdo a los datos tabulados se obtuvo que un $60 \%$ de los trabajadores refieren contar con protectores auditivos a disposición para su uso y tan solo un $40 \%$ manifestó no contar con dispositivos de protección auditiva a disposición inmediata para su uso. Sin embargo, el 90\% 
expreso su limitación para ellos escoger el tipo de protector auditivo a utilizar en la ejecución de sus tareas.

\section{Autoeficacia}

Un total de 18 trabajadores lo cual representa el 90\% expreso estar totalmente de acuerdo y/o de acuerdo en que no siempre pueden decidir cuándo necesitan usar protectores auditivos, a diferencia de un $10 \%$ lo cuales se sienten en capacidad de decidir cuándo deben hacerlo.

De igual manera se manifestó en las encuestas realizadas que un $80 \%$ saben cómo ajustar y utilizar los protectores auditivos, sin embargo, un $20 \%$ de ellos no se sienten en la capacidad de enseñarles la técnica a sus compañeros de trabajo.

Tabla 1. Distribución de los resultados de la encuesta aplicada $(n=20)$.

\begin{tabular}{|c|c|c|c|c|c|c|c|c|c|c|c|c|}
\hline & \multicolumn{2}{|c|}{ TA } & \multicolumn{2}{|c|}{ DA } & \multicolumn{2}{|c|}{ NA/ND } & \multicolumn{2}{|c|}{ D } & \multicolumn{2}{|c|}{ TD } & \multicolumn{2}{|c|}{$\mathrm{Ns} / \mathrm{Nc}$} \\
\hline & $\mathrm{n}$ & $\% n$ & $\mathrm{n}$ & $\% n$ & $\mathrm{n}$ & $\% n$ & $\mathrm{n}$ & $\% n$ & $\mathrm{n}$ & $\% n$ & $\mathrm{n}$ & \%n \\
\hline \multicolumn{13}{|c|}{ Susceptibilidad pérdida auditiva $^{a}$} \\
\hline Pregunta 1. & - & - & - & - & 2 & $10,0 \%$ & 6 & $30,0 \%$ & 10 & $50,0 \%$ & 2 & $10,0 \%$ \\
\hline Pregunta 14. & - & - & - & - & - & - & 12 & $60,0 \%$ & 8 & $40,0 \%$ & - & - \\
\hline \multicolumn{13}{|c|}{ Consecuencias pérdida auditiva $^{b}$} \\
\hline Pregunta 2. & 16 & $80,0 \%$ & 4 & $20,0 \%$ & - & - & - & - & - & - & - & - \\
\hline Pregunta 13. & 10 & $50,0 \%$ & 9 & $45,0 \%$ & 1 & $5,0 \%$ & - & - & - & - & - & - \\
\hline \multicolumn{13}{|c|}{ Beneficios acciones preventivas $^{c}$} \\
\hline Pregunta 5. & 1 & $5,0 \%$ & 15 & $75,0 \%$ & - & - & 4 & $20,0 \%$ & - & - & - & - \\
\hline Pregunta 16. & 7 & $35,0 \%$ & 7 & $35,0 \%$ & 6 & $30,0 \%$ & - & - & - & - & - & - \\
\hline Pregunta 24. & 5 & $25,0 \%$ & 12 & $60,0 \%$ & 3 & $15,0 \%$ & - & - & - & - & - & - \\
\hline \multicolumn{13}{|c|}{ Acción preventiva - comodidad $^{d}$} \\
\hline Pregunta 6. & 4 & $20,0 \%$ & 12 & $60,0 \%$ & 4 & $20,0 \%$ & - & - & - & - & - & - \\
\hline Pregunta 17. & 5 & $25,0 \%$ & 7 & $35,0 \%$ & 5 & $25,0 \%$ & 3 & $15,0 \%$ & - & - & - & - \\
\hline Pregunta 19. & - & - & 8 & $40,0 \%$ & 8 & $40,0 \%$ & 4 & $20,0 \%$ & - & - & - & - \\
\hline Pregunta 25. & 14 & $70,0 \%$ & 6 & $30,0 \%$ & - & - & - & - & - & - & - & - \\
\hline \multicolumn{13}{|c|}{ Acción preventiva - silenciar $^{e}$} \\
\hline Pregunta 7. & - & - & 7 & $35,0 \%$ & 2 & $10,0 \%$ & 11 & $55,0 \%$ & - & - & - & - \\
\hline Pregunta 8. & - & - & 4 & $20,0 \%$ & 5 & $25,0 \%$ & 7 & $35,0 \%$ & 3 & $15,0 \%$ & 1 & $5,0 \%$ \\
\hline Pregunta 18. & 6 & $30,0 \%$ & 11 & $55,0 \%$ & 3 & $15,0 \%$ & - & - & - & - & - & - \\
\hline Pregunta 26. & - & - & - & - & - & - & 9 & $45,0 \%$ & 11 & $55,0 \%$ & - & - \\
\hline \multicolumn{13}{|l|}{ Comportamientos $^{f}$} \\
\hline Pregunta 9. & 14 & $70,0 \%$ & 6 & $30,0 \%$ & - & - & - & - & - & - & - & - \\
\hline Pregunta 10. & - & - & - & - & 2 & $10,0 \%$ & 11 & $55,0 \%$ & 7 & $35,0 \%$ & - & - \\
\hline Pregunta 21. & 10 & $50,0 \%$ & 8 & $40,0 \%$ & 2 & $10,0 \%$ & - & - & - & - & - & - \\
\hline Pregunta 22. & - & - & 1 & $5,0 \%$ & 2 & $10,0 \%$ & 12 & $60,0 \%$ & 5 & $25,0 \%$ & - & - \\
\hline Pregunta 27. & - & - & 16 & $80,0 \%$ & 4 & $20,0 \%$ & - & - & - & - & - & - \\
\hline Pregunta 28. & 16 & $80,0 \%$ & 4 & $20,0 \%$ & - & - & - & - & - & - & - & - \\
\hline \multicolumn{13}{|l|}{ Normas sociales } \\
\hline Pregunta 3. & - & - & 12 & $60,0 \%$ & - & - & 8 & $40,0 \%$ & - & - & - & - \\
\hline Pregunta 11. & 8 & $40,0 \%$ & 8 & $40,0 \%$ & 1 & $5,0 \%$ & - & - & 1 & $5,0 \%$ & - & - \\
\hline Pregunta 20. & - & - & - & - & 2 & $10,0 \%$ & 18 & $90,0 \%$ & - & - & - & - \\
\hline \multicolumn{13}{|l|}{ Autoeficacia } \\
\hline Pregunta 4. & 4 & $20,0 \%$ & 14 & $70,0 \%$ & - & - & 2 & $10,0 \%$ & - & - & - & - \\
\hline Pregunta 12. & 7 & $35,0 \%$ & 9 & $45,0 \%$ & 4 & $20,0 \%$ & - & - & - & - & - & - \\
\hline Pregunta 15. & 4 & $20,0 \%$ & 9 & $45,0 \%$ & 5 & $25,0 \%$ & 2 & $10,0 \%$ & - & - & - & - \\
\hline Pregunta 23. & & - & 8 & $40,0 \%$ & 9 & $45,0 \%$ & 3 & $15,0 \%$ & - & - & - & - \\
\hline
\end{tabular}




\section{Discusión}

El establecer la percepción de los trabajadores ante el ruido industrial y los dispositivos de protección auditiva es un gran avance para implementar medidas preventivas y correctivas para evitar la hipoacusia en los trabajadores. Es de conocimiento público que la exposición sin un control al riesgo ocasiona enfermedades profesionales que derivan en pérdidas económicas para la empresa.

La percepción de los trabajadores con respecto al efecto que ocasiona el ruido industrial y el uso de equipos de protección auditivo dependen de un componente cognitivo, sin embargo, el uso de dichos equipos se ve condicionado por temas ergonómicos.

Independientemente del conocimiento que pudiese tener el trabajador con respecto a las consecuencias por la exposición al ruido, este no tendrá razones para tomar acciones preventivas en contra de dicho factor mientras su componente afectivo al ruido sea escaso (Contreras, 2013).

\section{Conclusiones}

El estudio de la percepción de ruido de los trabajadores evidencio que es necesario establecer un programa de conservación auditiva el cual abarque la capacitación a los trabajadores con respecto al uso, mantenimiento, sustitución y almacenamiento de los protectores auditivos.

Después de haber analizado los resultados antes expuestos se puede determinar que independientemente del conocimiento que el trabajador tenga con respecto a la relación entre la exposición al ruido excesivo y la perdida de la audición es necesario crear una real concientización del uso de protectores auditivos; una herramienta importante de mencionar para la prevención y concientización es el software "HLSlim" de la NIOSH, en el cual se simula una verdadera lesión en el sistema auditivo; lo cual permitiría promover la prevención de la pérdida auditiva y ayudaría sustancialmente a la concientización del uso de protectores auditivos.

Es necesario involucrar a todos los mandos de la empresa en la cultura de prevención para obtener su aprobación en las mejoras necesarias para el cuidado de la audición. Existen acciones de tipo administrativo que contrarrestan los puntos en los cuales los trabajadores manifiestan la incomodidad en el uso de protectores auditivos, siendo preciso analizar la posibilidad de disponer de varios modelos ergonómicos de dichos equipos. Adicionalmente sería adecuado incrementar señales lumínicas en los sitios de trabajo, donde el nivel de ruido supere los $85 \mathrm{~dB}$; con los cual los trabajadores no solo contaran con el sonido de la alarma de las maquinarias.

Es importante realizar estudios posteriores a la intervención preventiva para obtener una retroalimentación.

\section{Bibliografía}


Reddy RK, Welch D, Thorne P, Ameratunga S. Hearing protection use in manufacturing workers: Aqualitative study. Noise Health. 2012; 14(59): 202-209.

Gómez García AR, Espigares Rodríguez E, Suasnavas Bermúdez PR. La percepción del ruido industrial y su relación con las medidas preventivas en una industria textil del Ecuador Hig. Sanid. Ambient. 2013; 13(5):1097-1101.

Meliá Navarro JL. El factor humano en la seguridad laboral. Psicología de la Seguridad y Salud Laboral. Bilbao: Lettera Publicaciones; 2007.

Morillejo E A. La percepción del riesgo en la prevención de riesgos laborales. Apuntes de Psicología. 2002; 20(3): 415-426.

Actitudes Acerca de la Protección Auditiva y Pérdida de la Audición en Trabajadores de una Planta Compresora de Gas Costa-Afuera. Quevedo, Carlos AlbertoContreras.46,2013, Vol.15.07182449.

Svensson EB, Morata TC, Nylén P, Krieg EF, Johnson AC. Beliefs and attitudes among Swedish workers regarding the risk of hearing loss. Int J Audiol. 2004; 43(10):585-93.

Corrales M, Tovalín H, Rodríguez M. Percepción del Riesgo Sobre Protección y Pérdida Auditiva en Trabajadores Expuestos a Ruido en el Trabajo. Ciencia \& Trabajo. 2009; 11 (31): 1-4.

Contreras Quevedo CA. Actitudes Acerca de la Protección Auditiva y Pérdida de la Audición en Trabajadores de una Planta Compresora de Gas Costa-Afuera. Ciencia \& Trabajo. 2013; 15(46): 35-39.

Hannah K, Dhooge I, Degeest S, Vinck B. The effects of a hearing education program on recreational noise exposure, attitudes and beliefs toward noise, hearing loss, and hearing protector devices in young adults. Noise Health. 2015; 17(78): 253-262.

Keppler H, Dhooge I, Vinck B. Hearing in young adults. Part I: The effects of attitudes and beliefs toward noise, hearing loss, and hearing protector devices. Noise Health. 2015; 17(78):237244. 